This is an electronic reprint of the original article. This reprint may differ from the original in pagination and typographic detail.

Author(s): Gordon, Swanne; Lopez Sepulcre, Andres; Rumbo, Diana; Reznick, David N.

Title: $\quad$ Rapid Changes in the Sex Linkage of Male Coloration in Introduced Guppy Populations

Year: $\quad 2017$

Version:

Please cite the original version:

Gordon, S., Lopez Sepulcre, A., Rumbo, D., \& Reznick, D. N. (2017). Rapid Changes in the Sex Linkage of Male Coloration in Introduced Guppy Populations. American Naturalist, 189(2), 196-200. https://doi.org/10.1086/689864

All material supplied via JYX is protected by copyright and other intellectual property rights, and duplication or sale of all or part of any of the repository collections is not permitted, except that material may be duplicated by you for your research use or educational purposes in electronic or print form. You must obtain permission for any other use. Electronic or print copies may not be offered, whether for sale or otherwise to anyone who is not an authorised user. 
Note

\title{
Rapid Changes in the Sex Linkage of Male Coloration in Introduced Guppy Populations
}

\author{
Swanne P. Gordon, ${ }^{1, \star}, \dagger$ Andrés López-Sepulcre, ${ }^{1,2, \star}$ Diana Rumbo, ${ }^{3}$ and David N. Reznick ${ }^{3}$ \\ 1. Centre of Excellence in Biological Interactions, Department of Biological and Environmental Sciences, University of Jyväskylä, Jyväskylä, \\ Finland; 2. Centre National de la Recherche Scientifique Unité Mixte de Recherche (CNRS UMR) 7618, Institute of Ecology and \\ Environmental Sciences (iEES) Paris, Université Pierre et Marie Curie, Paris, France; 3. Department of Biology, University of California, \\ Riverside, California
}

Submitted August 12, 2016; Accepted October 7, 2016; Electronically published December 8, 2016

Online enhancements: appendix. Dryad data: http://dx.doi.org/10.5061/dryad.d30m0.

\begin{abstract}
AвSTRACT: Theory predicts that the sex linkage of sexually selected traits can influence the direction and rate of evolution and should itself evolve in response to sex-specific selection. Some studies have found intraspecific differences in sex linkage associated with differences in selection pressures, but we know nothing about how fast these differences can evolve. Here we show that introduced guppy populations showing rapid evolution of male coloration also show rapid changes in sex-linkage patterns. A comparison, using hormonal manipulations in females, of introduced populations of different ages suggests a consistent increase of autosomal or X-linked coloration 2 years after introduction from high- to low-predation environments. Twenty years after introduction, populations already show the same pattern of coloration inheritance typical of natural low-predation populations in similar habitats. These results highlight that the contemporary evolution of sexually selected traits ought to be studied in concert with contemporary changes in linkage relationships.
\end{abstract}

Keywords: Poecilia reticulata, rapid evolution, sex linkage.

\section{Introduction}

The mode of inheritance of sexually selected traits can strongly influence their rate and direction of evolution. This is because sex chromosomes and autosomes are subject to different sex-specific selection pressures (Fisher 1931). For example, while single recessive alleles are directly exposed to selection in the sex chromosomes of the hemizygous sex, they may be sheltered from selection when paired with dominant alleles in cases of autosomal or sex linkage in the homozygous sex (Charlesworth et al. 1987; Kirkpatrick

\footnotetext{
* Gordon and López-Sepulcre both served as lead authors for this project.

${ }^{\dagger}$ Corresponding author; e-mail: swanne.gordon@jyu.fi.

ORCIDs: López-Sepulcre, http://orcid.org/0000-0001-9708-0788; Rumbo, http://orcid.org/0000-0001-9122-1359.
}

Am. Nat. 2017. Vol. 189, pp. 196-200. (C) 2016 by The University of Chicago. 0003-0147/2017/18902-57171\$15.00. All rights reserved.

DOI: $10.1086 / 689864$ and Hall 2004). Strict Y linkage of male traits (where no recombination with the $\mathrm{X}$ occurs) may be favored in the case of sexual antagonism, where the trait is beneficial for males but detrimental to females. On the other hand, autosomal or X-linked traits may be selected when genetic correlations between male attractiveness and female preference are beneficial (Kirkpatrick and Hall 2004). Given this, changes in the nature and intensity of sexual selection may favor traits with one type of inheritance over the other in subsequent generations (Gordon et al. 2012).

Studies of traits undergoing contemporary evolution have proven instrumental in our understanding of natural selection and the genetic constraints on adaptation (Hendry and Kinnison 1999). Yet despite the above-mentioned theoretical expectations, these studies consider the mode of inheritance of sexually selected traits to be fixed. This implicitly assumes that changes in linkage relationships should be slow, compared to the evolution of the traits themselves. In this study we examine whether sex-linkage patterns can change rapidly during the evolution of male coloration in experimental introductions of the Trinidadian guppy Poecilia reticulata.

Male guppies originating from high-predation populations are consistently less colorful than those from low-predation populations (Endler 1980; Houde 1997; Magurran 2005). This has been attributed to a selective advantage for crypsis in high-predation environments (Endler 1980) versus the predominance of female preference for conspicuous and novel color patterns in low-predation environments (Magurran 2005). Phylogenetic comparisons show that low-predation populations descend from multiple independent evolutionary colonizations of high-predation populations into the upstream reaches of each river (Alexander et al. 2006; Suk and Neff 2009). Moreover, experimental introductions of high-predation guppies into upstream low-predation environments triggered rapid evolutionary change toward more 
colorful males in as few as 2 years (approx. 6 generations; Endler 1980; Kemp et al. 2009).

Guppies have Y chromosomes with a large pseudoautosomal region (PAR) that recombines with the $\mathrm{X}$ chromosome, and a nonrecombining region (male-specific Y [MSY] region) associated with a sex-determining locus (Nanda et al. 1992, 2014; Traut and Wilking 2001). Twenty-four guppy color genes are known to be linked to the PAR, 16 are strictly $\mathrm{Y}$ linked (on the MSY region), two are strictly X linked (in the region homologous to the MSY), and two are autosomal (Haskins et al. 1961; Lindholm and Breden 2002). Although every adult male in both low- and high-predation environments shows a variety of color patterns, females have no color patterns. However, male coloration can be inherited but unexpressed in females if autosomal or X linked. The expression of these genes can be induced through hormonal manipulation (see appendix, available online).

Previous studies using hormone assays and pedigree crossings (Gordon et al. 2012) show that the linkage of male coloration varies with predation pressure and sexual selection. Specifically, male guppies from natural high-predation populations display paternally inherited coloration (i.e., strictly Y linked) more often than their low-predation counterparts (Haskins et al. 1961). This trend is consistent with theory predicting that stronger sexual selection (in low-predation environments; Houde 1997) favors female inheritance through indirect benefits (i.e., the production of "sexy sons") and the evolution of a genetic correlation between female preference and attractive male traits (Lande 1981; Kirkpatrick and Hall 2004).

Despite this relationship between linkage and sexual selection, it remains unclear whether the fast evolution of male coloration observed in experimental introductions is associated with equally rapid changes in sex linkage. To exam- ine this, we take advantage of several past experiments, performed in different years, in which guppies were introduced from high-predation to low-predation populations, allowing us to reconstruct a time line of changes in trait sex linkage down to a resolution of 1 year (approx. 3 generations). These results could have important consequences for the study of sexual selection and the rapid evolution of sexually dimorphic traits.

\section{Methods}

The study includes guppies from eight populations. Six of them were populations experimentally introduced from high-predation (HP) to guppy-free low-predation (LP) environments between 1 and 54 years (3-170 generations) before sampling. Two populations were the natural ancestral populations (Aripo and Guanapo; HP) from which the introduced fish were derived. All experimental introductions were performed to study the evolution of male coloration and/or life histories (see references in table 1). In each experiment, guppies ( $n=70-300)$ from a natural HP environment were translocated into upstream LP tributaries that initially contained no resident guppies. All introduction sites were upstream of waterfalls that had historically excluded both predators and guppies. The experimental introductions analyzed in this study were initiated between 1957 and 2009 (table 1), but all were sampled in 2009 and 2010. Therefore, they allowed us to reconstruct a time line of evolutionary changes based on the duration of the introduction: more recent introductions represented earlier stages in the adaptation process to the novel LP environments than older introductions.

All introductions resulted in rapid evolution of increased male coloration and other traits after 1-10 years. One of the

Table 1: Description of samples used in this study

\begin{tabular}{|c|c|c|c|c|c|c|c|c|}
\hline Collection & Type & $\begin{array}{c}\text { Introduction } \\
\text { year }\end{array}$ & $\begin{array}{l}\text { Collection } \\
\text { year }\end{array}$ & $\begin{array}{l}\text { Population } \\
\text { age (years) }\end{array}$ & Generations & Rearing & $n$ & Reference \\
\hline \multicolumn{9}{|l|}{ Aripo: } \\
\hline Aripo HP & Natural & $\ldots$ & 2009 & 0 & 0 & Wild & 23 & \\
\hline Endler & Introduced & 1976 & 2009 & 28 & $48-93$ & Wild & 15 & Endler 1980 \\
\hline \multicolumn{9}{|l|}{ Guanapo: } \\
\hline Guanapo HP W & Natural & $\ldots$ & 2009 & 0 & 0 & Wild & 20 & \\
\hline Guanapo HP CG1 & Natural & $\ldots$ & 2009 & 0 & 0 & $\mathrm{~F}_{2}$ & 11 & \\
\hline Guanapo HP CG2 & Natural & $\ldots$ & 2010 & 0 & 0 & $\mathrm{~F}_{2}$ & 10 & \\
\hline Caigual & Introduced & 2009 & 2010 & 1 & 3 & $\mathrm{~F}_{2}$ & 10 & Travis et al. 2014 \\
\hline Lower La Laja & Introduced & 2008 & 2009 & 1 & 3 & $\mathrm{~F}_{2}$ & 16 & Travis et al. 2014 \\
\hline Upper La Laja 1 & Introduced & 2008 & 2009 & 1 & 3 & $\mathrm{~F}_{2}$ & 10 & Travis et al. 2014 \\
\hline Upper La Laja 2 & Introduced & 2008 & 2010 & 2 & $4-7$ & $\mathrm{~F}_{2}$ & 20 & Travis et al. 2014 \\
\hline El Cedro & Introduced & 1981 & 2009 & 23 & $40-76$ & Wild & 14 & Kemp et al. 2009 \\
\hline Turure $\mathrm{LP}^{\mathrm{a}}$ & Introduced & 1957 & 2009 & 52 & $88-172$ & Wild & 18 & Haskins et al. 1961 \\
\hline
\end{tabular}

Note: $\mathrm{HP}=$ high predation; $\mathrm{LP}=$ low predation.

${ }^{a}$ Although the source population for this introduction was Guanapo HP, the Turure stream forms part of the Oropuche drainage. 
8 introduced LP populations (Upper La Laja) was sampled in two consecutive years, thus providing two different time points. Because of logistical constraints, some experiments were performed on wild-caught individuals while others were performed on second-generation descendants of wild-caught fish reared in common-garden conditions. Guanapo HP was sampled in both 2009 and 2010, with 2009 including both wild-caught and common-garden samples. Table 1 describes the sampling and introduction details of the analyzed populations. Results for natural Aripo and Guanapo LP localities from Gordon et al. (2012) are included in figure 1 for reference but were not part of our analysis here.

Exposure of females to testosterone causes them to express male color genes not contained in the MSY region (since this region is present only in males; Haskins et al. 1961; Gordon et al. 2012). Because very few color loci in guppies are autosomal and the majority of these are rarely found in wild populations (Lindholm and Breden 2002), the appearance of color in a treated female is strongly suggestive of $\mathrm{X}$ linkage (which can be in either the PAR or the region homologous to the MSY), yet our results by themselves cannot rule out the contribution of autosomal linkage. Hormonal manipulation of females has been shown to correlate with pedigree assessments of sex linkage in male guppies (Haskins et al. 1961). During the assay, each female was housed individually in a 2-gallon glass aquarium. We treated every tank with $100 \mu \mathrm{L}$ of alpha methyl-testosterone diluted in $95 \%$ ethanol $(1 \mathrm{mg} / \mathrm{mL})$ every 3 days. Each aquarium was cleaned and had approximately $50 \%$ of its water changed every 15 days, before the addition of a dose of testosterone. Each trial ran for approximately 60 days of testosterone treatment, which is sufficient for a complete expression of all color genes (following Gordon et al. 2012). We took digital photographs of each fish before, during, and after the treatment under a light source that closely mimicked the spectrum of natural sunlight (BlueMax full-spectrum bulb, Jackson, MI). Structural coloration (green, blue, purple, silver), which is not readily seen in photographs, was not included in analyses. A single individual assessed color presence from the digital pictures by using a color standard that was present in all photographs. All such assessments were "blind," meaning that they were done without knowledge of the origin of the fish. We recorded the presence/absence of melanistic and carotenoid coloration on the body as well as the presence of any coloration on the tail after 2 months of testosterone treatment. We used presence/absence of given colors as our response variables. Assuming that our females bear a random sample of (non-Y-linked) color patterns shown in wild males, the proportion of females expressing color under testosterone provides an estimate for the proportion of patterns that are not strictly Y linked. Implicitly, the interpretation of an increase in female expression of color as a relative increase of non-Y linkage also assumes that all adult males show the color in question (melanistic, carotenoid, and tail), or at least that the color presence in males did not increase after introduction. To confirm the case, we scored pic-
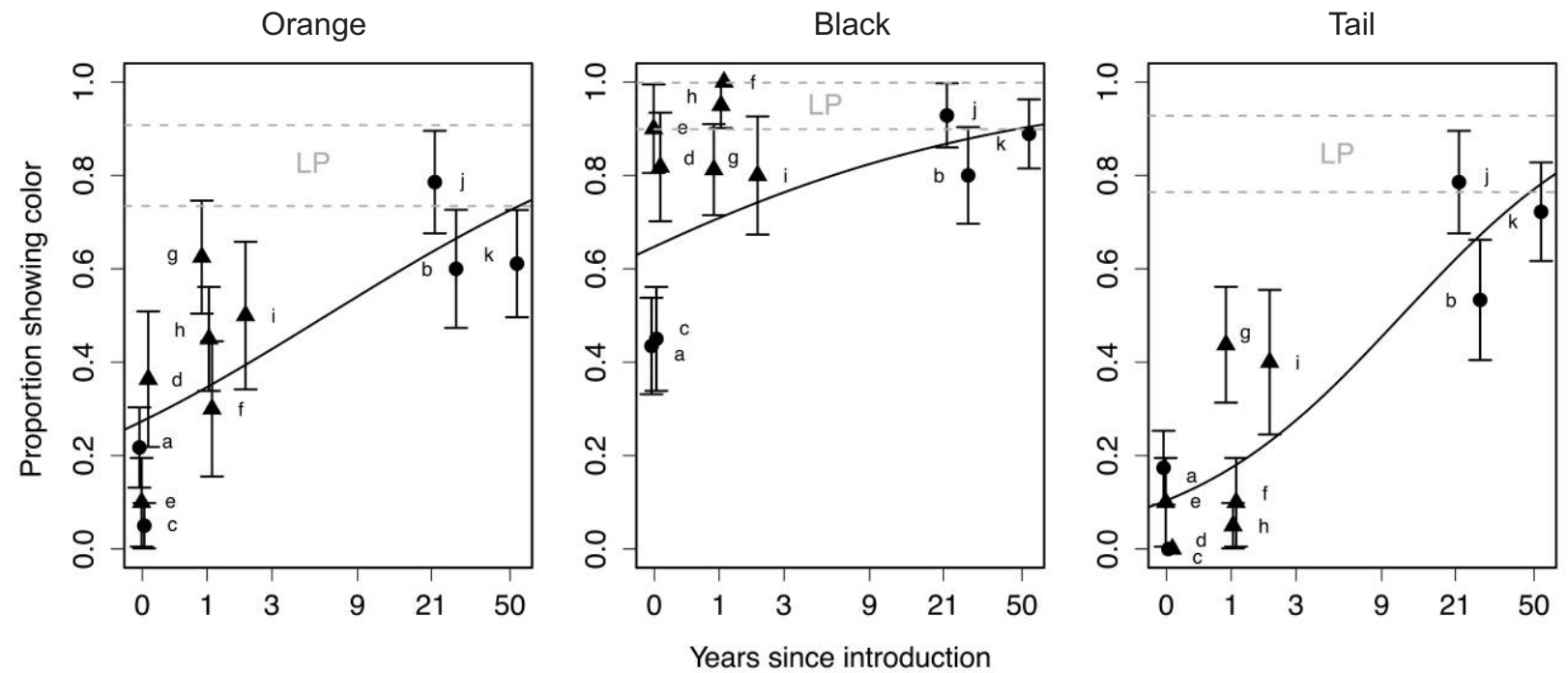

Figure 1: Proportion of testosterone-treated females showing coloration as a function of introduction age. Error bars denote proportion standard errors. Solid lines show the model fit. The grayed area displays two standard deviations for natural Guanapo and Aripo low-predation (LP) populations combined (from Gordon et al. 2012). Triangles denote lab $\mathrm{F}_{2}$ samples and circles wild-caught individuals. Points associated with a 0 value on the $X$-axis are the high-predation (HP) ancestral populations. The letters beside the points refer to the populations in table 1 , as follows: $\mathrm{a}=$ Aripo HP; $\mathrm{b}=$ Endler; $\mathrm{c}=$ Guanapo HP W; $\mathrm{d}=$ Guanapo HP CG1; e = Guanapo HP CG2; f = Caigual; $\mathrm{g}=$ Lower La Laja; $\mathrm{h}=$ Upper La Laja 1; $\mathrm{i}=$ Upper La Laja 2; $\mathrm{j}=$ El Cedro; $\mathrm{k}=$ Turure LP. 
tures of wild-caught males from all study populations (sample sizes ranging between 25 and 92 individuals). Consistent with the assumption, $100 \%$ of mature males showed melanistic and carotenoidcoloration. Tailcoloration was also present in 100\% of males from all populations except Upper La Laja $(99 \%, n=$ $92)$ and El Cedro $(85.3 \%, n=34)$. Given that the only detectable difference was a decrease in the presence of tail coloration 21 years after introduction, our interpretation is justified.

We analyzed the proportion of females showing a given color by fitting generalized linear mixed models with a binomial response and a logit link in $\mathrm{R}$, version 3.1.2, as a function of time since introduction. We took account of differences in rearing conditions (wild/common garden) by including wild caught versus lab reared as a fixed effect. Drainage and the interaction between introduction age and rearing were also tested but were then excluded because their contribution was not significant (all $P>.1$ ). River of origin (Guanapo HP and Upper La Laja) was included as a random effect. The data used in this analysis are available in the Dryad Digital Repository: http://dx.doi.org/10.5061/dryad.d30m0 (Gordon et al. 2017).

\section{Results}

The presence of orange in females showed a significant increase with the duration of the introduction (fig. 1; estimate $=0.049 \pm 0.018, Z=2.69, P<.007)$. Rearing environment was not significant for orange coloration (estimate $=1.004 \pm 0.581, Z=1.726, P=.084)$. We observed a significant but moderate response to introduction age for black coloration (fig. 1; estimate $=0.056 \pm 0.015$, $Z=4.57, P<.001)$ and a significant effect of lab rearing (estimate $=2.09 \pm 0.46, Z=4.57, P<.001$ ). The strongest introduction age effect was for tail coloration (fig. 1; estimate $=0.88 \pm 0.17, Z=5.14, P<.001)$, which did not show an effect of lab rearing (estimate $=0.315 \pm 0.795$, $Z=0.39, P=.692)$.

\section{Discussion}

Previous studies showed that when guppy populations are exposed to relaxed predation through experimental introductions, males evolve increased coloration in as little as 3 years, or approximately 6-9 generations (Endler 1980; Kemp et al. 2009). Here we show, for the first time, that these changes are associated with similarly rapid changes in the linkage of male coloration. This is particularly true for orange and tail coloration, which are known to be important for mate choice (Houde 1997). Specifically, as guppy populations adapt to low-predation environments they show an increase in autosomal or X-linked male color (a higher proportion of females bearing the trait), compared to their high-predation ancestral populations (few females bearing the trait). These results are consistent with similar differences in linkage relationships observed between natural lowpredation populations and their high-predation ancestors (Gordon et al. 2012). Although it took 20 years (34-66 generations) for these patterns of linkage to be comparable to those in natural low-predation populations, sizable changes occurred within 2 years (4-7 generations; fig. 1).

We were surprised to find that lab-reared females showed coloration more often than wild ones. However, this difference was significant only for black coloration, known to respond plastically to environmental conditions (Houde 1997).

We can think of three non-mutually exclusive genetic mechanisms behind the rapid changes in the degree of sex linkage as high-predation guppies adapt to low-predation environments (see appendix for details). First, these changes could reflect increases in the frequencies of non-MSY-linked color variants that were kept at low frequencies in the source populations. In other words, the change in linkage patterns is due to increased sexual selection on standing variation of recombining color alleles. However, if the changes observed were simply due to a single locus (or few loci) of large effect, our results could have occurred through selective processes neutral to linkage. While quantitative trait loci analyses show that color patterns in guppies are controlled by multiple loci of small effect in natural populations (Tripathi et al. 2009), we do not yet have trait maps for experimental populations. Therefore, we cannot completely discard this possibility.

Second, the results could represent new autosomal or $\mathrm{X}$-linked mutations. However, given the rate of the observed changes here and the rarity of mutations, this seems unlikely to explain a large extent of the results.

A third possibility is that the increase in the proportion of females carrying male-coloration loci in the novel lowpredation populations is due to the crossover of alleles from the nonrecombining MSY to the PAR. This could be caused by the evolution of recombination within the MSY region. There is one example of such change in guppies. The male color allele $(s b)$ was found to be strictly Y linked (MSY) in one ancestral high-predation population yet inherited on either the $\mathrm{X}$ or the $\mathrm{Y}$ chromosome (PAR) in its natural lowpredation counterpart (Lindholm and Breden 2002). If this mechanism is driving our results, it has very important consequences for the evolution of recombination and sexchromosome differentiation. More research is needed to evaluate this possibility.

Although the particular genetic mechanism(s) behind our results cannot be revealed by our study, the trends suggest some interesting and consistent population patterns. Populations exposed to lower levels of predation and higher levels of sexual selection show a rapid increase in the degree of autosomal and/or X-linked coloration. This increase in the importance of female inheritance can arise through 
a variety of selective mechanisms. Selection for increased coloration in low-predation environments may favor genes with two allele copies (linked to the PAR or autosomes), which can produce more conspicuous coloration through increased gene dosage (Farr 1983). Also, increased sexual selection may drive the evolution of a genetic correlation between female preference and male coloration, which is not conducive to strict Y linkage (Kirkpatrick and Hall 2004).

Regardless of the selective and genetic forces behind it, our findings reveal an increase in the proportion of females that are carriers of male color variation, which has important implications for the evolution of sexually selected traits through indirect effects (Kirkpatrick and Hall 2004). Moreover, the speed of the changes observed suggests a dynamic interaction between the evolution of traits and their sex linkage, and it also suggests that studies on the evolution of sexually selected traits should consider contemporary changes in its sex-linkage relationships.

\section{Acknowledgments}

We would like to thank the undergraduate research interns of the Reznick Lab for help with rearing and maintaining the fish populations used in this study. All research for this specific experiment was carried out in accordance with the approved animal care and use protocol at the University of California, Riverside (AUP-20080008). Export permits were kindly granted by the Fisheries Division of Trinidad and Tobago, with invaluable support from I. Ramnarine. Funding was provided by the National Science Foundation (Frontiers for Integrative Biological Research grant EF 0623632 to D.N.R.), Le Fonds de Recherche du Québec-Nature et Technologies (postgraduate scholarship to S.P.G.), and the Academy of Finland (Postdoctoral Fellowship project 2100002744 to S.P.G.).

\section{Literature Cited}

Alexander, H. J., J. S. Taylor, S. S. T. Wu, and F. Breden. 2006. Parallel evolution and vicariance in the guppy (Poecilia reticulata) over multiple spatial and temporal scales. Evolution 60:2352-2369.

Charlesworth, B., J. A. Coyne, and N. H. Barton. 1987. The relative rates of evolution of sex-chromosomes and autosomes. American Naturalist 130:113-146.

Endler, J. 1980. Natural selection on color patterns in Poecilia reticulata. Evolution 34:76-91.

Farr, J. A. 1983. The inheritance of quantitative fitness traits in guppies, Poecilia reticulata (Pisces: Poeciliidae). Evolution 37:1193-1209.

Fisher, R. 1931. The evolution of dominance. Biological Reviews 6:345368.
Gordon, S. P., A. López-Sepulcre, and D. N. Reznick. 2012. Predationassociated differences in sex linkage of wild guppy coloration. Evolution 66:912-918.

Gordon, S. P., A. López-Sepulcre, D. Rumbo, and D. N. Reznick. 2017. Data from: Rapid changes in the sex linkage of male coloration in introduced guppy populations. American Naturalist, Dryad Digital Repository, http://dx.doi.org/10.5061/dryad.d30m0.

Haskins, C. P., E. F. Haskins, J. J. A. McLaughlin, and R. E. Hewitt. 1961. Polymorphism and population structure in Lebistes reticulates. Pages 320-395 in W. F. Blair, ed. Vertebrate speciation. University of Texas Press, Austin.

Hendry, A. P., and M. T. Kinnison. 1999. The pace of modern life: measuring rates of contemporary microevolution. Evolution 53:16371653.

Houde, A. E. 1997. Sex, color, and mate choice in guppies. Princeton University Press, Princeton NJ.

Kemp, D. J., D. N. Reznick, G. F. Grether, and J. A. Endler. 2009. Predicting the direction of ornament evolution in Trinidadian guppies (Poecilia reticulata). Proceedings of the Roval Society B 276: $4335-4343$.

Kirkpatrick, M., and D. Hall. 2004. Sexual selection and sex linkage. Evolution 58:683-691.

Lande, R. 1981. Models of speciation by sexual selection on polygenic traits. Proceedings of the National Academy of Sciences of the USA 78:3721-3725.

Lindholm, A., and F. Breden. 2002. Sex chromosomes and sexual selection in poeciliid fishes. American Naturalist 160(suppl.):S215S224.

Magurran, A. E. 2005. Evolutionary ecology: the Trinidadian guppy. Oxford University Press, New York.

Nanda, I., M. Schartl, W. Feichtinger, J. T. Epplen, and M. Schmid. 1992. Early stages of sex chromosome differentiation in fish as analyzed by simple repetitive DNA sequences. Chromosoma 101:301310.

Nanda, I., S. Schories, N. Tripathi, C. Dreyer, T. Haaf, M. Schmid, and M. Schartl. 2014. Sex chromosome polymorphism in guppies. Chromosoma 123:373-383. doi:10.1007/s00412-014-0455-z.

Suk, H. Y., and B. D. Neff. 2009. Microsatellite genetic differentiation among populations of the Trinidadian guppy. Heredity 102: $425-434$.

Traut, W., and H. Wilking. 2001. Meiotic chromosomes and stages of sex chromosome evolution in fish: zebrafish, platyfish, and guppy. Chromosome Research 9:659-672.

Travis, J., D. N. Reznick, R. D. Bassar, A. López-Sepulcre, R. Ferrière, and T. Coulson. 2014. Do eco-evo feedbacks help us understand nature? answers from studies of the Trinidadian guppy. Advances in Ecological Research 50:1-40.

Tripathi, N., M. Hoffmann, E. M. Willing, C. Lanz, D. Weigel, and C. Dreyer. 2009. Genetic linkage map of the guppy, Poecilia reticulata, and quantitative trait loci analysis of male size and colour variation. Proceedings of the Roval Societv B 276:2195-2208.

Associate Editor: Charles F. Baer Editor: Yannis Michalakis 